International Journal of Pure and Applied Mathematics

Volume 90 No. $4 \quad 2014,433-438$

ISSN: 1311-8080 (printed version); ISSN: 1314-3395 (on-line version)

url: http://www.ijpam.eu

doi: http://dx.doi.org/10.12732/ijpam.v90i4.5

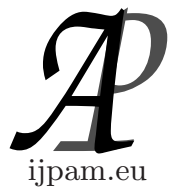

\title{
A NOTE ON THE COMPUTATION OF INCIDENCE MATRICES OF SIMPLICIAL COMPLEXES
}

\author{
Veerendra Vikram Awasthi \\ Department of Mathematics and Statistics \\ Indian Institute of Science Education and Research - Kolkata \\ Mohanpur, West Bengal, 741 252, INDIA
}

\begin{abstract}
Incidence Matrices have been defined only for graphs. In this article we present a method to compute the incidence matrices for simplicial complexes.
\end{abstract}

AMS Subject Classification: 05B20, 05C50, 05E45, 35J05

Key Words: incidence number, incidence matrix, simplicial complex

\section{Introduction}

H. Poincaré specifically emphsized the application of incidence matrices in his article [3]. Thereafter, Incidence Matrices are being used as a tool from linear algebra to derive important properties of long and complicated graphs. In this article, we generalise the notion of (vertex-edge) incidence matrix to $\left(\sigma^{n}-\sigma^{n-1}\right)$ incidence matrix where $\sigma^{n-1}$ and $\sigma^{n}$ are $n-1$ and $n$ dimensional simplexes of an orientated simplicial complex. We define these incidence matrices by using a concept from algebraic topology called incidence number [1]. In this article we give an alternative formula using incidence numbers. The entries of these incidence matrices are the incidence numbers of oriented simplexes which differ

Received: August 28, 2013

(c) 2014 Academic Publications, Ltd. url: www.acadpubl.eu 
by 1 in their dimensions. We show that the alternative formula is equivalent to the definition of the (vertex-edge) incidence matrix of a graph as defined in (Chapter 2, [2]) . The importance of this formula is that it can be generalised to higher diemensions of directed graph i.e., oriented simplicial complex.

\section{Incidence Number}

Definition 1. [1] The incidence number associated with two simplexes which differ by 1 in their dimension $\left(\sigma^{p+1}, \sigma^{p}\right)$ of an oriented simplicial complex $K$ denoted by $\left[\sigma^{p+1}, \sigma^{p}\right]$ is defiend as follows:

(a) If $\sigma^{p}$ is not a face of $\sigma^{p+1}$, we put $\left[\sigma^{p+1}, \sigma^{p}\right]=0$.

(b) If $\sigma^{p}$ is a face of $\sigma^{p+1}$, This means all the vertices of $\sigma^{p}$ are also the vertices of $\sigma^{p+1}$ except one vertex as $\sigma^{p+1}$ has $p+2$ vertices and $\sigma^{p}$ has $p+1$ vertices. Let us label the vertices of $\sigma^{p}$ so that $\left\langle v_{0}, v_{1}, \cdots, v_{p}\right\rangle$ gets positive orientation. Let $v$ be the additional vertex of $\sigma^{p+1}$. Then putting $v$ as the first vertex $\left\langle v, v_{0}, \cdots, v_{p}\right\rangle$, we get either $\sigma^{p+1}$ is positively oriented or negatively oriented. We define

$$
\left[\sigma^{p+1}, \sigma^{p}\right]= \begin{cases}1 & \text { if }<v, v_{0}, \cdots, v_{p}>=+\sigma^{p+1} \\ -1 & \text { if }<v, v_{0}, \cdots, v_{p}>=-\sigma^{p+1} .\end{cases}
$$

The above definition says that the incidence number $\left[\sigma^{p+1}, \sigma^{p}\right]$ of two simplexes where one is the face of other and their dimensions differ by 1 is either +1 or -1 depending on the orientation $\sigma^{p+1}$ gets by putting the additional vertex at first order. If $\sigma^{p+1}$ gets positive orientation then the incidence number is +1 or else -1 .

Example 2.1. Let $K$ the closure of $\sigma^{2}$, where $\sigma^{2}=<v_{0}, v_{1}, v_{2}>$, be oriented by the ordering $v_{0}<v_{1}<v_{2}$. Then the incidence numbers of simplices can be calculated as follows.

$$
\begin{array}{cc}
{\left[\sigma^{2},<v_{0}, v_{1}>\right]=+1,} & {\left[<v_{0}, v_{1}>,<v_{1}>\right]=+1,} \\
{\left[\sigma^{2},<v_{1}, v_{2}>\right]=+1,} & {\left[<v_{0}, v_{1}>,<v_{0}>\right]=-1,} \\
{\left[\sigma^{2},<v_{0}, v_{2}>\right]=-1,} & {\left[<v_{0}, v_{1}>,<v_{2}>\right]=0 .}
\end{array}
$$




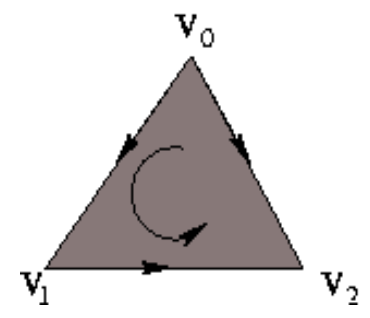

\section{Incidence Matrix of a Graph}

A directed graph can be viewed as an oriented 1-dimensional simplicial complex with edges as 1-simplexes and the vertices as 0 -simplexes, In this section we prove that an alternative formula can be given to the definition of incidence matrix of a graph which can be generalised to the higher dimensional oriented simplicial complexes.

The incidence matrix of a directed graph is is defined as follows:

Definition 2. [2] The incidence matrix of a directed graph $G$ is a $p \times q$ matrix $\left(a_{i j}\right)$ where $p$ and $q$ are the number of vertices and edges respectively, such that $a_{i j}=-1$ if the edge $e_{j}$ leaves vertex $v_{i}, \quad\left(a_{i j}\right)=1$ if the edge $e_{j}$ enters the vertex $v_{i}$ and 0 otherwise.

Let us consider the following example:

Example 3.1. Consider the directed graph $G$ shown in figure 3.1, with $V=\left\{v_{1}, v_{2}, v_{3}, v_{4}\right\}$ and $E=\left\{e_{1}, e_{2}, e_{3}, e_{4}, e_{5}\right\}$. The incidence matrix of $G$ is given by

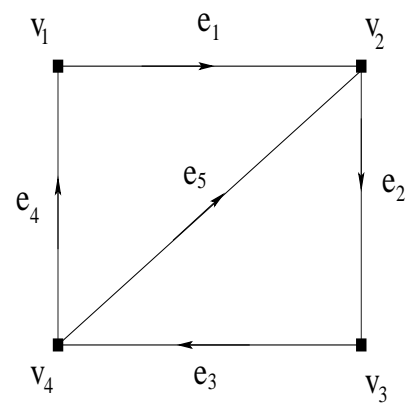

Figure 3.1: Directed Graph $G$ 


$$
E=\left(\begin{array}{ccccc}
-1 & 0 & 0 & 1 & 0 \\
1 & -1 & 0 & 0 & 1 \\
0 & 1 & -1 & 0 & 0 \\
0 & 0 & 1 & -1 & -1
\end{array}\right)
$$

Notations. As a directed graph as 1-dimensional oriented simplicial complex. We denote the edges as 1-simplexes i.e. $\left\{\sigma_{1}^{1}, \sigma_{2}^{1} \cdots \sigma_{n}^{1}\right\}$ and all the vertex as 0 -simplexes namely, $\left\{\sigma_{0}^{0}, \sigma_{1}^{0} \cdots \sigma_{m}^{0}\right\}$. We now prove the following Theorem.

Theorem 3. The incidence matrix of a directed graph $G$ with $m$ vertices and $n$ edges is the following matrix

$$
I_{G}=\left(a_{i j}\right)_{m \times n}
$$

where $a_{i j}$ is the incidence number $\left[\sigma_{j}^{1}, \sigma_{i}^{0}\right]$. Where $\sigma_{j}^{1}$ is the $\mathrm{j}^{\text {th }}$ directed edge and $\sigma_{i}^{0}$ is $\mathrm{i}^{\text {th }}$ vertex.

Proof. Let us lebel all the edges and vertices of the graph as given in notation abve. If any two vertex are non-adjacent i.e., they are not connected by any edge then their incidence number is zero. Let the 1 -simplex $\sigma_{j}^{1}$ be any arbitrary edge joining the vertices $\sigma_{i}^{0}$ and $\sigma_{j}^{0}$. If the edge $\sigma_{j}^{1}$ leaves the vertex $\sigma_{i}^{0}$ and enters the vertex $\sigma_{j}^{0}$ then the edge $\sigma_{j}^{1}=<\sigma_{i}^{0}, \sigma_{j}^{0}>$ gets the orientation as $\sigma_{i}^{0}<\sigma_{j}^{0}$.

Calculating the incidence numbers $\left[\sigma_{j}^{1}, \sigma_{i}^{0}\right]$ and $\left[\sigma_{j}^{1}, \sigma_{j}^{0}\right]$ we get $\left[\sigma_{j}^{1}, \sigma_{i}^{0}\right]=-1$ which is same as $a_{i j}$ by Definition 3.1 and $\left[\sigma_{j}^{1}, \sigma_{j}^{0}\right]=1=a_{j j}$. Thus the formula given in the statement of the theorem is equivalent to the definition of the incidence matrix.

Example 3.2. Consider the directed graph $G$ as in Example 3.1. We calculate the incidence matrix of $G$ by using incidence number. By above Theorem we get

$$
\begin{gathered}
E=\left(\begin{array}{lllll}
{\left[e_{1}, v_{1}\right]} & {\left[e_{2}, v_{1}\right]} & {\left[e_{3}, v_{1}\right]} & {\left[e_{4}, v_{1}\right]} & {\left[e_{5}, v_{1}\right]} \\
{\left[e_{1}, v_{2}\right]} & {\left[e_{2}, v_{2}\right]} & {\left[e_{3}, v_{2}\right]} & {\left[e_{4}, v_{2}\right]} & {\left[e_{5}, v_{2}\right]} \\
{\left[e_{1}, v_{3}\right]} & {\left[e_{2}, v_{3}\right]} & {\left[e_{3}, v_{3}\right]} & {\left[e_{4}, v_{3}\right]} & {\left[e_{5}, v_{3}\right]} \\
{\left[e_{1}, v_{4}\right]} & {\left[e_{2}, v_{4}\right]} & {\left[e_{3}, v_{4}\right]} & {\left[e_{4}, v_{4}\right]} & {\left[e_{5}, v_{4}\right]}
\end{array}\right) \\
E=\left(\begin{array}{ccccc}
-1 & 0 & 0 & 1 & 0 \\
1 & -1 & 0 & 0 & 1 \\
0 & 1 & -1 & 0 & 0 \\
0 & 0 & 1 & -1 & -1
\end{array}\right)
\end{gathered}
$$

Thus both the defintions are equivalent. 


\section{Incidence Matrices of Oriented Simplicial Complex}

In this section, we generalize the formula obtained for computing the incidence matrix of a graph in Theorem 3.1 to the oriented simplicial complex $K$.

Definition 4. Let $K$ be a oriented simplicial complex of dimension $k$. Let the total number of $k-1$ simplexes be $m$ and the total number of $k$ simplexes be $n$ Then incidence matrix of order $k, I_{k}$ is defined as follows:

$$
I_{k}=M_{m \times n}=\left(a_{i j}\right)_{m \times n} .
$$

Here

$$
a_{i j}=\left[\sigma_{j}^{k}, \sigma_{i}^{k-1}\right] \quad \text { for } i=1, \cdots, m, j=1, \cdots, n,
$$

and $\left[\sigma_{j}^{k}, \sigma_{i}^{k-1}\right]=$ the incidence number of pair $\left(\sigma_{j}^{k}, \sigma_{i}^{k-1}\right)$.

Consider the following example.

Example 4.1. Let $K$ be the following simplicial complex We observe that the dimension of $K$ is 2 as the largest simplex it has is a 2-simplex namely, $\left.<v_{0}, v_{1}, v_{2}\right\rangle$. In the simplicial complex $K$ we note that $K$ has only one 2 simplex, four 1-simplex and four 0 -simplex. We give an orientation to $K$ as follows $v_{0}<v_{1}<v_{2}<v_{3}$.

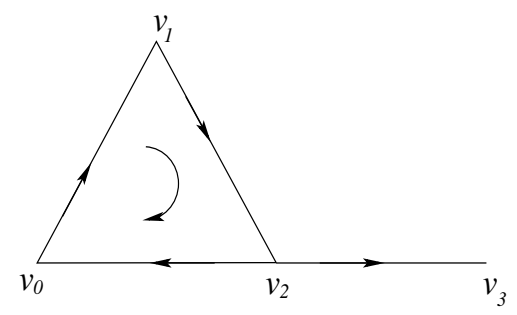

Simplicial Complex $K$

First we define the incidence Matrix $I_{2}$ as the matrix $M_{m \times n}$ where $n$ is the number of 2-simplices i.e. $n=1$ and $m$ is the number of 1 -simplices i.e., $m=4$. Thus in this example the incidence matrix $I_{2}$ is the Matrix $M_{4 \times 1}$.

We name all the simplices as follows:

$$
\begin{aligned}
\sigma^{2} & =<v_{0}, v_{1}, v_{2}>, \\
\sigma_{1}^{1} & =<v_{0}, v_{1}>,
\end{aligned}
$$




$$
\begin{aligned}
\sigma_{2}^{1} & =\left\langle v_{1}, v_{2}\right\rangle \\
\sigma_{3}^{1} & =\left\langle v_{2}, v_{3}\right\rangle \\
\sigma_{4}^{1} & =\left\langle v_{2}, v_{0}\right\rangle .
\end{aligned}
$$

Now we define $I_{2}$ as follows:

$$
I_{2}=M_{4 \times 1}=\left(\begin{array}{l}
{\left[\sigma^{2}, \sigma_{1}^{1}\right]} \\
{\left[\sigma^{2}, \sigma_{2}^{1}\right]} \\
{\left[\sigma^{2}, \sigma_{3}^{1}\right]} \\
{\left[\sigma^{2}, \sigma_{4}^{1}\right]}
\end{array}\right)=\left(\begin{array}{l}
{\left[<v_{0}, v_{1}, v_{2}>,<v_{0}, v_{1}>\right]} \\
{\left[<v_{0}, v_{1}, v_{2}>,<v_{1}, v_{2}>\right]} \\
{\left[<v_{0}, v_{1}, v_{2}>,<v_{2}, v_{3}>\right]} \\
{\left[<v_{0}, v_{1}, v_{2}>,<v_{2}, v_{0}>\right]}
\end{array}\right)=\left(\begin{array}{l}
1 \\
1 \\
0 \\
1
\end{array}\right) .
$$

Here $\left[\sigma^{2}, \sigma_{i}^{1}\right]$ denotes the incidence number of the pair $\left(\sigma^{2}, \sigma_{i}^{1}\right)$.

Similarly, we will define the incidence matrix $I_{1}$. We find that in $K$ there are four 1-simplex and four 0-simplex. Therefor by above argument the order of the incidence matrix $I_{1}$ will be $4 \times 4$. Proceeding same as above, we get

$$
\begin{array}{r}
I_{1}=M_{4 \times 4}=\left(\begin{array}{rrrr}
{\left[\sigma_{1}^{1}, \sigma_{1}^{0}\right]} & {\left[\sigma_{2}^{1}, \sigma_{1}^{0}\right]} & {\left[\sigma_{3}^{1}, \sigma_{1}^{0}\right]} & {\left[\sigma_{4}^{1}, \sigma_{1}^{0}\right]} \\
{\left[\sigma_{1}^{1}, \sigma_{2}^{0}\right]} & {\left[\sigma_{2}^{1}, \sigma_{2}^{0}\right]} & {\left[\sigma_{3}^{1}, \sigma_{2}^{0}\right]} & {\left[\sigma_{4}^{1}, \sigma_{2}^{0}\right]} \\
{\left[\sigma_{1}^{1}, \sigma_{3}^{0}\right]} & {\left[\sigma_{2}^{1}, \sigma_{3}^{0}\right]} & {\left[\sigma_{3}^{1}, \sigma_{3}^{0}\right]} & {\left[\sigma_{4}^{1}, \sigma_{3}^{0}\right]} \\
{\left[\sigma_{1}^{1}, \sigma_{4}^{0}\right]} & {\left[\sigma_{2}^{1}, \sigma_{4}^{0}\right]} & {\left[\sigma_{3}^{1}, \sigma_{4}^{0}\right]} & {\left[\sigma_{4}^{1}, \sigma_{4}^{0}\right]}
\end{array}\right) \\
=\left(\begin{array}{ccccc}
-1 & 0 & 0 & 1 \\
1 & -1 & 0 & 0 \\
0 & 1 & -1 & -1 \\
0 & 0 & 1 & 0
\end{array}\right) .
\end{array}
$$

Thus we have an alternative formula for computing the incidence matrix of directed graphs which is generalised to compute the incidence matrices of higher orders for an oriented simplicial complex.

\section{References}

[1] Satya Deo, Algebraic Topology. A Primer, Hindustan Book Agency, India (2003).

[2] A. Kaveh, Optimal Analysis of Structures by Concepts of Symmetry and Regularity, Springer-Verlag, Wien (2013).

[3] H. Poincaré, Second complément à l'Analysis Situs, Proc. London Math. Soc., 32 (1900), 277-308. 